\title{
Simulation of wave propagation in flexible hoses
}

\author{
R. Etlender ${ }^{1}$, U. Iben ${ }^{1} \&$ M. Bischoff ${ }^{2}$ \\ ${ }^{1}$ Robert Bosch GmbH, Department CR/ARH, Stuttgart, Germany \\ ${ }^{2}$ University of Stuttgart, Institute of Structural Mechanics, \\ Stuttgart, Germany
}

\begin{abstract}
A fluid-structure interaction model is presented for transient flow in flexible hoses. The wall shear stress is included. The governing equations for the fluid flow are discretized by a finite-volume method. The wall is considered as a thin structure consisting of shells and is discretized by a finite element method. The coupling of the solvers is applied iteratively-staged.

Keywords: quasi one-dimensional conservation laws, wave propagation, fluidstructure interaction, Godunov method, cavitation.
\end{abstract}

\section{Introduction}

The development of complex hydraulic systems, such as fuel injection systems, requires efficient simulation models to analyze system properties. Wave propagations in flexible hoses have a significant influence on both the transient mass flow rate over specific parts and the overall system behavior. Due to the restriction of computational time, quasi one-dimensional models have to be used for system simulations.

In industrial applications flexible hoses are not only used due to their low cost but also to damp pressure oscillations. To simulate the overall system behavior, the common frequency analysis based on linear transfer matrices can not be used. This is due to the following facts: firstly, the occurring pressure waves have high amplitudes, therefore linear transfer properties are not valid anymore. Secondly, the simulation tools applied in the development process use time based algorithms instead of frequency domain. Thus to simulate the fluid structure interaction in the hoses, algorithms using a compressible flow solver and a solver for the structure of the flexible pipes have to be developed. 
In the presented work compressible fluid flow is considered as quasi onedimensional flow with constant entropy. The conservation of mass and momentum, including a weak variable cross sectional pipe area $A$, are considered. The higher order terms (viscous terms) of the Navier-Stokes equation are replaced by approximations derived from semi-empirical models. In order to secure the conservation properties a finite volume scheme for $A \rho$ and $A \rho v$ is developed. Cavitation induced by pressure waves in the pipes can be handled due to the fully conservative approach. The wall is considered as a thin structure and methods from finite-element analysis of shells are used for the discretization. To increase the efficiency of the numerical method the dimension of the wave equation for the wall motion is reduced by a degeneration approach. The complete numerical model consists of a combination of a fluid and a structure solver in each time step guaranteeing the conservation properties of the flow equations.

The fluid-structure-model, the numerical method and results are presented with emphasis on the analysis of wave propagation for different hydraulic boundary conditions.

\section{Physical model}

Let us consider an infinitesimal section of the hose with interior cross-section area $A=A(x, t)$. It is filled with compressible fluid with density $\rho$. The crosssection average velocity is denoted by $v(x, t)$ and the pressure by $p(x, t) . x$ and $t$ mark the co-ordinate along the pipe and the time respectively. Furthermore, let $\tau_{W}=\tau_{W}(x, t)$ denote the wall shear stress of the hose in the given section. The entropy of the fluid is considered to be constant, so that the energy equation can be neglected. Furthermore, the fluid flow is considered isothermal, so that the density is a function of the pressure and the temperature of the fluid at the beginning of the simulation only, i.e. $\rho=\rho\left(p, T_{0}\right)$. This assumption is legitimate for flexible hoses, as they neither are used in high-pressure region, nor subject to greater and fast temperature fluctuations.

Regard conservation of mass for the pipe section and assume $\rho, v$ and $A$ to be smooth in time and space. The continuity equation of a fluid flow in the hose can be specified:

$$
\frac{\partial}{\partial t}(A \rho)+\frac{\partial}{\partial x}(A \rho v)=0 .
$$

Regarding conservation of momentum with the same restrictions, the equation of motion for one-dimensional flow can be specified:

$$
\frac{\partial}{\partial t}(A \rho v)+\frac{\partial}{\partial x}\left(A \rho v^{2}\right)+A \frac{\partial}{\partial x} p=A \tau_{W} .
$$

To make the system of equations complete, a state equation $p=p\left(\rho, T_{0}\right)$ and an equation for the wall motion are needed. The recent shows the interrelationship between the forces acting on the wall and the changing of the cross-section area. It can be either a simple dependence of $A$ on $p$ or any more complex model up to multidimensional wave equation. The efficient modeling of the wall, as well as 
its numerical handling and the effects on the coupling algorithm, will be discussed later. Assuming a presence of the missing equation and a method for its numerical handling, a conservative numerical scheme for discretization of the fluid equation along with a coupling scheme is targeted in this work first.

As the form of the continuous equations is essential for the conservation properties of its discrete versions, consider two slightly recasted formulations of the system of equations before constructing a finite-volume-scheme. Using differentiation by parts technique following equivalent formulations can be generated:

$$
\begin{aligned}
& \frac{\partial}{\partial t}(\rho)+\frac{\partial}{\partial x}(\rho v)=-\frac{\rho}{A}\left(\frac{\partial}{\partial t} A+v \frac{\partial}{\partial x} A\right) \\
& \frac{\partial}{\partial t}(\rho v)+\frac{\partial}{\partial x}\left(\rho v^{2}+p\right)=-\frac{\rho v}{A}\left(\frac{\partial}{\partial t} A+v \frac{\partial}{\partial x} A\right)+A \tau_{M}
\end{aligned}
$$

and

$$
\begin{aligned}
& \frac{\partial}{\partial t}(A \rho)+\frac{\partial}{\partial x}(A \rho v)=0 \\
& \frac{\partial}{\partial t}(A \rho v)+\frac{\partial}{\partial x}\left(A \rho v^{2}+p\right)=p \frac{\partial}{\partial x} A+A \tau_{M} .
\end{aligned}
$$

The different formulations of the conservation laws represent different views of the consequence of wall motions for the fluid states. The variables $A \rho$ and $A \rho v$ are observed as conservative in the equation (4). The equation (3) treats the density $\rho$ and the momentum $\rho v$ as conservative variables (like the equations for one-dimensional flows in inelastic pipes) and considers the wall motion as source therms.

To construct a model for $\tau_{M}$ some known semi-empirical models for the friction in inelastic pipes can be used. Appearing constants of the models have to be validated by experiments.

Invoking Brunone et al. [5] the wall shear stress can be written in following form:

$$
\tau_{M}=-\frac{f}{2} \rho v^{2}+\frac{k}{4} \rho A\left(\frac{\partial}{\partial t} v-c \frac{\partial}{\partial x} v\right)
$$

where $f$ is the steady-state coefficient (Darcy-Weisbach friction factor), $k$ the unsteady friction coefficient and $c$ the water hammer wave velocity. The authors specify $k$ as a function of the Reynolds number $R e$. A similar model was provided by Axworthy et al. [1]. For the wall shear stress they deduced:

$$
\tau_{M}=-\frac{f}{2} \rho v^{2}+\frac{\tilde{k}}{4} \rho A\left(\frac{\partial}{\partial t} v+v \frac{\partial}{\partial x} v\right)
$$

where, unlike the previous model, $\tilde{k}$ is a time and space dependent coefficient which represents the frequency. 
A substantially different approach was provided by Zielke [11]. It is based on frequency-dependent friction analysis and supplies the following formula for the wall shear stress:

$$
\tau_{M}=-\frac{4 \nu}{r} \rho v+\frac{2 \nu}{r} \int_{0}^{t} W_{d}(t-\tilde{t}) \frac{\partial(\rho v)}{\partial \tilde{t}} d \tilde{t}
$$

where $W_{d}$ are some weight functions, with which the bygone values of flow rate variation are appraised according to their meaning to the present pressure loss in the pipe section. Note, that due to the advanced approach by Theissen [9], it is possible to eliminate the imperative integration over the whole past simulation time $\left(t_{0}, t_{\text {now }}\right)$ and to construct an efficient numerical method for friction calculations. This makes Zielke's model especially attractive for quasi onedimensional calculations. Detailed analysis of this particular friction model is omitted here, for further information, see $[9,11]$.

\section{Discretization of conservation equations}

As our main objective is to guarantee the conservation properties of the discrete numerical scheme for a full time step involving a solver for the fluid states, a solver for the structure states and its coupling, a finite-volume-scheme for the fluid equations is constructed, following Godunov's idea. It is tempting to start with the formulation (3), use the common Godunov-methods (for inelastic pipes) to approach the left side and apply the right side to the discrete scheme by using the source-splitting technique. However, considering the hose to be divided in $N$ finite sections, i.e. $\Delta x=x_{i+1}-x_{i}, x_{i+\frac{1}{2}}=x_{i}+\frac{\Delta x}{2}$. The time is discretized according to CFL-condition with $\Delta t=t^{n+1}-t^{n}, t^{n+\frac{1}{2}}=t^{n}+\frac{\Delta t}{2}$. The mass and the momentum conservations can only be provided in this case by ensuring the sum over all source terms to be 0 at every time step $\left[t_{n}, t_{n+1}\right]$ :

$$
\sum_{i=1}^{N}\left[\frac{\rho}{A}\left(\frac{\partial}{\partial t} A+v \frac{\partial}{\partial x} A\right)\right]_{i}^{n+\frac{1}{2}}=0, \quad \sum_{i=1}^{N}\left[\frac{\rho v}{A}\left(\frac{\partial}{\partial t} A+v \frac{\partial}{\partial x} A\right)\right]_{i}^{n+\frac{1}{2}}=0
$$

where $[\cdot]_{i}^{n}$ denotes a term evaluated at the point $i$ and at time $n$. Neither solving of a wave equation, nor using simple models for the computing of cross-section variations enforce a priori balancing of sources, which are caused by prolongations and constrictions of the pipe. An analysis of the balancing of the source terms is omitted here, as it often tends to produce instabilities in the coupling algorithm and as the construction of a conservative discrete equation starting with (4) is straightforward.

Integrating the equation (4) over a finite cell $\left[x_{i-\frac{1}{2}}, x_{i+\frac{1}{2}}\right]$ and over a time slice $\left[t_{n}, t_{n+1}\right]$, using the trapezoidal rule to approach $\iint p \frac{\partial}{\partial x} A d x d t$ and the midpoint rule to approach every other integral, the following discrete scheme for a finite 
time step can be generated:

$$
\begin{aligned}
A_{i}^{n+1} \rho_{i}^{n+1}=A_{i}^{n} \rho_{i}^{n} & +\frac{\Delta t}{\Delta x}\left(A_{i-\frac{1}{2}}^{n+\frac{1}{2}}[\rho v]_{i-\frac{1}{2}}^{n+\frac{1}{2}}-A_{i+\frac{1}{2}}^{n+\frac{1}{2}}[\rho v]_{i+\frac{1}{2}}^{n+\frac{1}{2}}\right) \\
A_{i}^{n+1}[\rho v]_{i}^{n+1}=A_{i}^{n}[\rho v]_{i}^{n} & +\frac{\Delta t}{\Delta x}\left(A_{i-\frac{1}{2}}^{n+\frac{1}{2}}\left[\rho v^{2}+p\right]_{i-\frac{1}{2}}^{n+\frac{1}{2}}-A_{i+\frac{1}{2}}^{n+\frac{1}{2}}\left[\rho v^{2}+p\right]_{i+\frac{1}{2}}^{n+\frac{1}{2}}\right) \\
& +\frac{\Delta t}{2 \cdot \Delta x}\left(p_{i+\frac{1}{2}}^{n+\frac{1}{2}}\left(A_{i+1}^{n+\frac{1}{2}}-A_{i}^{n+\frac{1}{2}}\right)+p_{i-\frac{1}{2}}^{n+\frac{1}{2}}\left(A_{i}^{n+\frac{1}{2}}-A_{i-1}^{n+\frac{1}{2}}\right)\right) \\
& +\Delta t\left[A \tau_{M}\right]_{i}^{n+\frac{1}{2}}
\end{aligned}
$$

For approximation of the fluid state values on cell boundaries at half a time step, reconstruction-evaluation technique can be applied along with an appropriate Riemann-solver. (c.f. [7, 10]). Construction of the cross-section values depends on the model used for the pipe wall simulation.

\section{Simulation of the pipe wall motion}

The simplest and calculation-friendliest way to cover the cross-section variation in the simulation is to assume that the current prolongation depends only on the current pressure within the pipe. This view presumes the wall to be massless and to have no self-induced motion. Though this approximation is only inaccurate, the model is a good sample for studying the properties of the finite-volume scheme (for the flow equations) and the coupling algorithm, as it allows the cross-section area to be unsteady in time and space and tends much more than the others to cause instabilities in the complete algorithm. In fact, it presents the limit behavior of most complex models.

Let $R$ denote the interior radius of the pipe, $E$ the Young's bulk modulus of the wall material, $\mu$ the contraction rate of the wall, $s_{W}$ the section thickness and $w$ the wall displacement in the radial direction. This simple model induces the formula

$$
w=\frac{R^{2}\left(1-\mu^{2}\right)}{s_{W} \cdot E} \cdot p
$$

for a hose with clamped ends. Some more detailed models can be constructed by assuming the wall to be a thin structure and treating a wall section as being consisted on shell elements. According to the shell theory, the motion can be described by parameterizing shells and "degenerating" a multidimensional equation to a system of one-dimensional equations: assuming that a single shell has $M$ degrees of freedom, one shell can be represented by $M$ variables. As the shells are tangent to each other in only one dimension, one obtains $M$ one-dimensional equations to describe the wall motion. Figure 1 illustrates some multi-parameter models for a hose wall section.

Let us focus on $M=2$ element. This is the simplest model, which considers the longitudinal and transversal vibrations of the hose wall and is one, which does not require practical analysis of the interplay of the composites of the wall. This 


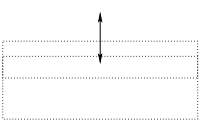

$M=1$

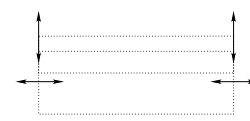

$M=2$

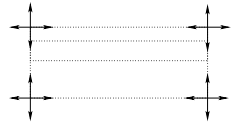

$M=4$

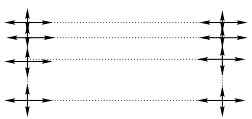

$M=8$

Figure 1: Possible parameterizations of a hose wall shell.

model requires only the knowledge of the "average" Young's modulus in radial and in axial directions (let $E_{\phi}$ and $E_{x}$ denote these values), of the "average" density of the wall material $\left(\rho_{W}\right)$ and of the "average" contraction rates $\left(\mu_{\phi}, \mu_{x}\right)$. Let radial and axial displacements, denoted by $w, u$ respectively, be the shell describing parameters (taking this two of all results in a vivid wave equation), with the upper notations for fluid states one obtains:

$$
\left\{\begin{aligned}
\frac{\partial^{2}}{\partial x^{2}} u+C_{1} \frac{\partial}{\partial x} w-C_{2} \frac{\partial^{2}}{\partial t^{2}} u & =F_{1}(t, x) \\
C_{3} w+\frac{\partial}{\partial x} u+C_{4} \frac{\partial^{2}}{\partial t^{2}} w & =F_{2}(t, x) .
\end{aligned}\right.
$$

with

$$
\begin{aligned}
& C_{1} \quad:=\frac{\mu_{\phi}}{R}, \quad C_{2} \quad:=\frac{\rho_{M}\left(1-\mu_{\phi} \mu_{x}\right)}{E}, \quad F_{1}(t, x) \quad:=\rho \tau_{W}, \\
& C_{3} \quad:=\frac{1}{R \mu_{x}}, \quad C_{4} \quad:=\frac{\rho_{M}\left(1-\mu_{\phi} \mu_{x}\right)}{E \mu_{x}}, \quad F_{2}(t, x) \quad:=\frac{R\left(1-\mu_{\phi} \mu_{x}\right)}{s E \mu_{x}} \cdot p(t, x) .
\end{aligned}
$$

\section{Fluid-structure coupling scheme}

For constructing a complete scheme for execution of a time step a coupling scheme is needed. As the fluid solver requires structure data from the end of the current time step and the structure solver requires fluid states from the end of the current step, it is impossible to use the parallel-coupling technique. Instead, iterativestaged algorithm has to be constructed. The chosen start for the iteration is the following: The fluid equations for fixed structure (for current time step) are solved as a predictor. The adapting of structure is done in the next step. This procedure is the starting point of the iteration fluid $\rightarrow$ structure $\rightarrow$ fluid $\rightarrow$ structure $\rightarrow \ldots$ which calculates improving approximations for the values at the end of the time step. The iteration is determined when the greatest change in the pressure values, created by one iteration round, is smaller than a (user-defined) accuracy bound. The next time step can be applied. To ensure the convergence of this iteration, a relaxation is required. Instead of using the new approximations for the wall, the combination of the old structure values and the currently approximated ones, weighted with the relaxation factor $\alpha$ and $(1-\alpha)$ respectively, is provided to the fluid solver in every step. For the information on finding the optimal relaxation factor [8] is referred. 


\section{Numerical results}

The scheme was implemented in $\mathrm{C}$ and integrated in a commercial hydraulic system simulation tool. The following calculations were done on a Pentium 3, $3 \mathrm{GHz}$ machine. The simulated time was $10 \mathrm{~ms}$ and the simulations took between 10 and $15 \mathrm{~s}$.

One important factor in wave propagation simulation is handling the pressure shocks. To present the consequence of wall flexibility on shocks, the behavior of a connection between two tanks with different pressures (10 bar and 12 bar) after opening the separating valve is discussed. (c.f. Figure 2)

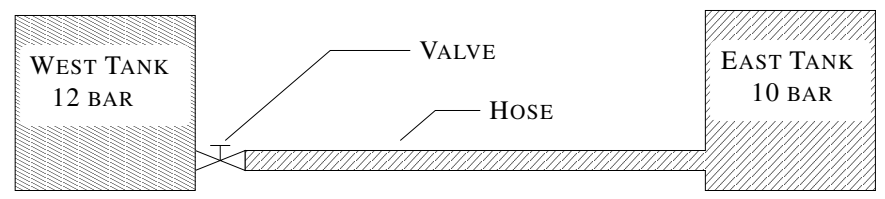

Figure 2: Test configuration.

Let us imagine the following setup: the hose has the length $l=0.5 m$, the interior diameter $d=0.003 \mathrm{~m}$, the contraction number $\mu=0.45$ and the wall thickness $s_{w}=0.002 \mathrm{~m}$. We compare a hose with Young's modulus $E_{x}=E_{\phi}=$ $5 \cdot 10^{8} \mathrm{~N} / \mathrm{m}^{2}$ with one with $E_{x}=E_{\phi}=5 \cdot 10^{9} \mathrm{~N} / \mathrm{m}^{2}$ and with an inelastic pipe. For the calculation the frequency-dependent friction model was used along with the $M=2$ model for the wall motion calculation. Figure 3 shows the pressure at the midpoint of the hoses and the pipe and Figure 4 flow rates at the valve.

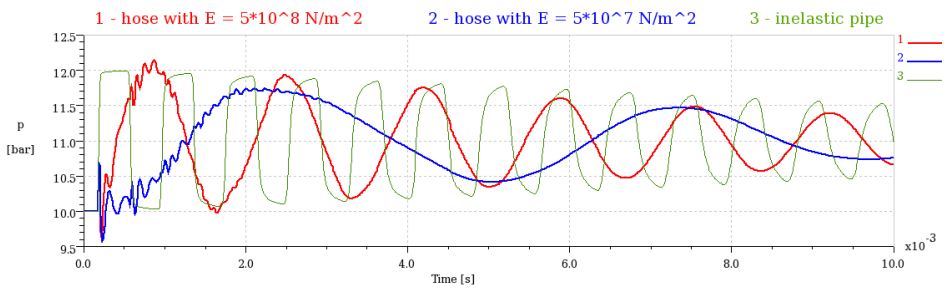

Figure 3: Pressure at the midpoint of the pipeline.

Both diagrams show, that rising flexibility causes rising delays in propagation of shocks. Furthermore, both hoses show stronger damping properties than the inelastic pipe.

The effect of using different models for the wall motion are shown in Figure 5. Note a basic problem of the hose modeling: wall flexibility causes strong changes on the propagation speed. Small errors in modeling cause therefore bigger discrepancies with progressing time. 
138 Fluid Structure Interaction and Moving Boundary Problems IV

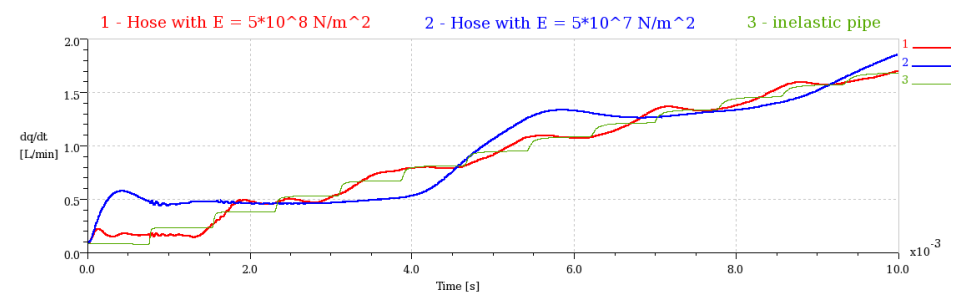

Figure 4: Flow rate at the valve.

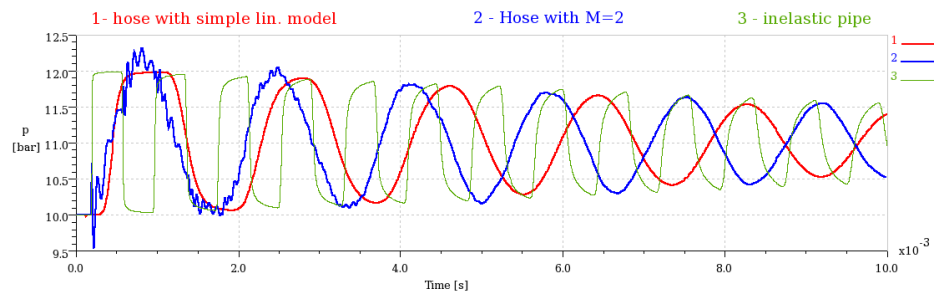

Figure 5: Pressure at the midpoint of the pipeline.

Let us also take a look at cavitation handling by the scheme. Barotrop model is used to describe cavitation, c.f. [2]. Imagine the west tank to be vacuumed in the same setup as before. Figure 6 shows the pressure, observed at the midpoints of the pipelines. The solution stays stable and again the same damping properties can be noticed.

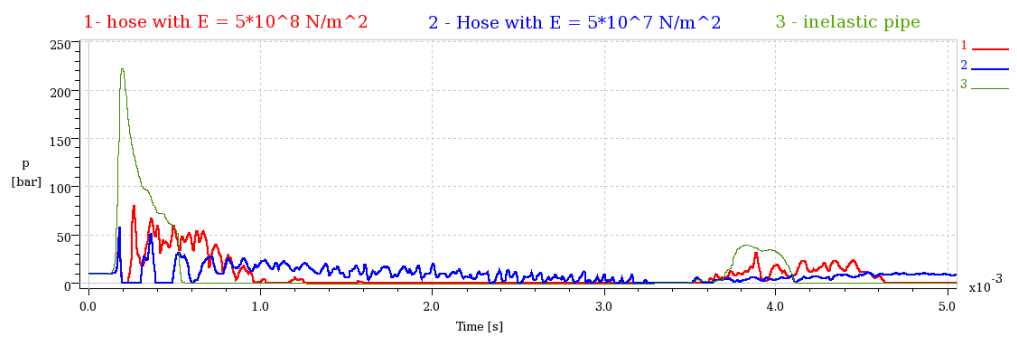

Figure 6: Pressure at the midpoint for a vacuumed west tank.

\section{Conclusions}

This paper sketches a method for efficient computation of wave propagation in hydraulic hoses for using it as a sub-model for simulation of complex hydraulic systems. The wall flexibility is included and the conservation properties 
are fulfilled in the discrete scheme. Due to using a finite-volume scheme for calculating fluid states, the algorithm has shown no problems with handling cavitation. Any new constructed model for the wall motion can be easily integrated in the complete scheme.

\section{Acknowledgement}

We would like to thank our colleagues A. Berg and M. Jungemann for fruitful discussions and hints.

\section{References}

[1] D. H. Axworthy, M. S. Ghidaoui, and D. A. McInnis. Extended thermodynamics derivation of energy dissipation in unsteady pipe flow. J. of Hydraulic Engineering, 126:276-287, 2000.

[2] M. Beck. Modelierung und Simulation der Wellenbewegung in kavitierenden Hydraulikleitungen. $\mathrm{PhD}$ thesis, 2003.

[3] M. Bischoff. Theorie und Numerik einer dreidimensionalen Schalenformulierung. PhD thesis, University of Stuttgart, 1999.

[4] D. Braes. Finite Elemente. Springer, 2002.

[5] B. Brunone, U.M. Golia, and M.Greco. Some remarks on the momentum equation for fast transients. In Proc. Int. Conf. on Hydraulic Transients with Water Column Separationg, 1991.

[6] H. K. Iben and U. Iben. Starthilfe Strömungslehre. B.G.Teubner, 1999.

[7] D. Kröner. Numerical Schemes for Conservations Laws. Wiley, Teubner, 1997.

[8] D. P. Mok. Partitionierte Lösungsansätze in der Strukturdynamik und der Fluid-Struktur-Interaktion. PhD thesis, University of Stuttgart, 2003.

[9] H. Theissen. Berücksichtigug instatinärer Rohrströmung bei der Simulation hydraulischer Anlagen. PhD thesis, RWTH Aachen, 1983.

[10] E. F. Toro. Riemann Solvers and Numerical Methods for Fluid Dynamics. A Practical Introduction. Springer, 1999.

[11] W. Zielke. Frequency-dependent friction in transient pipe flow. J. of Basic Engineering, 90:109-115, 1968. 